\title{
Anticuerpos Monoclonales como Terapia Antineoplásica
}

\author{
FRANKLIN ALDECOA \\ Médico Oncólogo Clínico. Profesor Asociado de Inmunología, Facultad de Medicina de la \\ Universidad San Martin de Porres. Grupo Peruano de Estudio de la Biología Molecular en Cáncer.
}

\section{ESTRUCTURA Y PRODUCCIÓN DE LOS ANTICUERPOS MONOCLONALES}

Los anticuerpos están compuestos por cadenas de proteínas con una conformación especial en forma de $\mathrm{Y}$, en la cual se puede identificar dos cadenas pesadas idénticas $(H)$ y dos cadenas ligeras idénticas (L) unidas por enlaces disulfuro intra e intercatenarias. Cada cadena liene dominios estructurales, los cuales son formaciones globulares derivadas de la conformación terciaria proteica a esos niveles: dos en las cadenas ligeras (uno constante $C_{L}$, y otro variable $V_{L}$ ).

Las cadenas pesadas contienen un dominio variable $\left(V_{11}\right)$ y 3 ó 4 dominios constantes $\left(C_{H}\right)$, dependiendo del isotipo del anticuerpo. La IgG está compuesta por 3 dominios constantes con la región bisagra (porción más flexible de toda la estructura) ubicada entre $\mathrm{C}_{\mathrm{H}} \perp$ y $\mathrm{C}_{\mathrm{H}} 2$, lugar donde la papaína actúa escindiendo la molécula en tres partes: dos porciones Fab (Fragment antigen-binding) en relación al reconocimiento antigénico y una porción Fc (Fragment crystalline) en relación a ligazón con receptores de la superficie celular y a la fijación del complemento. La especificidad y afinidad del receptor del antígeno en la región aminoterminal está determinada por 6 áreas hipervariables ( 3 de la cadena $\mathrm{H}$ y 3 de la $\mathrm{L}$ ), las cuales son llamadas determinantes antigénicos 0 regiones determinantes de complementariedad $\left({ }^{1-4}\right)$ (Figura 1). La diversidad (repertorio de anticuerpos) se produce en el ámbito genécico por recombinación somática a partir de una misma línea germinal, postulado probado por Susumo

Correspondencia:

Dr. Franklin Aldecon Bedoya

Pablo Carriquiry 179. Dpto. 201. Corpac

San Isidro. Lima 27 - Perú

e-mail:franklin.aldecoa@roche.com
Tonegawa, por cuyo trabajo obtuvo el Premio Nobel en $1987\left({ }^{5}\right)$.

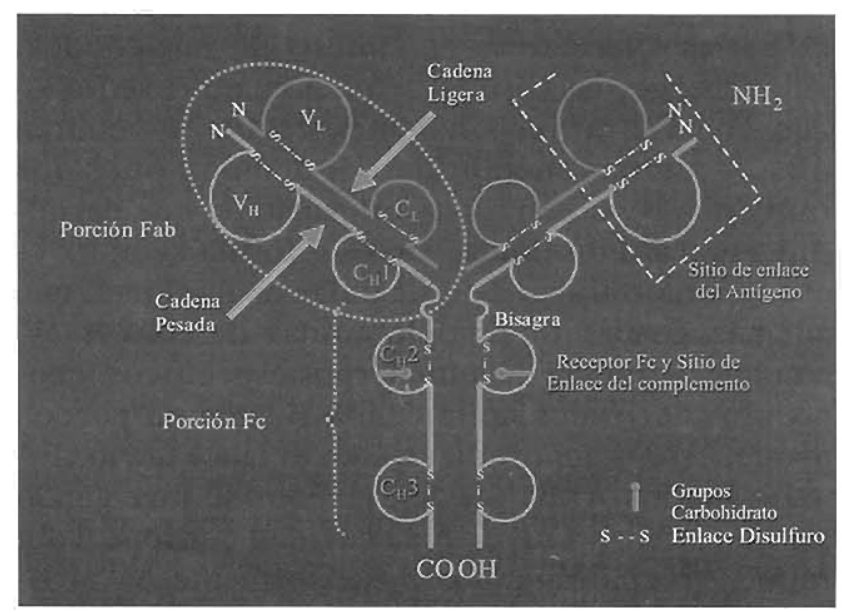

Figura 1.- Estructura de las inmunoglobulinas. E) diagrama corresponde a la inmunoglobulina $G$ : ticne 3 dominios constantes en la cadena pesada $\mathrm{y}$ la región bisagra entre $\mathrm{C}_{11} 1 \mathrm{y} \mathrm{C}_{11} 2$.

La mayoría de anticuerpos monoclonales ( $A M C$ ) son de origen murino y producidos por una combinación híbrida derivada de la fusión de una línea celular de mieloma inmortal, con una célula $\mathrm{B}$ productora de un anticuerpo específico conocido, a partir de un ratón inmunizado con células enteras, extractos celulares o antígenos purificados. Las técnicas utilizadas para poder fusionar ambas células incluyen el uso del virus Sendai (originalmente usado por Köhler y Milstein en 1975 en el origen del hibridoma, lo cual les permitió ganar el Premio Nobel en 1984) $\left({ }^{6}\right)$, el uso del polietilenglicol y de corriente electrica. Debido a que la fusión es un evento relarivamente raro cuando se usa las dos primeras técricas, se utiliza una estrategia de selección para asegurar el 
crecimiento de las células fusionadas o hibridoma e inhibir el crecimiento de las células de mieloma que no lograron unirse al correspondiente linfocito $\mathrm{B}$. Basados en estos hechos, se usa una línea celular de mieloma con deficiencia en la formación de la enzima hipoxantina-guanina fosforribosil-transferasa o la timidina quinasa, importantes en las vías metabólicas de rescate en la biosíntesis de purinas y pirimidinas. Por tanto, al usar un bloqueador de las vías de síntesis de nucleótidos -como la aminopterina- para formar ácidos nucleicos, las células de mieloma que no se fusionen morirán, al no tener la posibilidad de usar vías alternativas de síntesis de nucleótidos; sin embargo, las células de mieloma que se fusionen con las correspondientes células $B$ tendrán dicho camino metabólico indemne, pues los linfocitos $\mathrm{B}$ poseen dicha información que permitirá vivir al hibridoma. De esta forma se aprovecha la inmortalidad de las células de mieloma, que además genera inmunoglobulinas desconocidas, con la generación de anticuerpos específicos y conocidos derivados de la célula $B$, que no pueden mantenerse o inmortalizarse en el laboratorio. Finalmente los AMC pueden elaborarse inyectando los hibridomas a nivel de la cavidad peritoneal del ratón y recolectándolos posteriormente en el líquido ascítico correspondiente, o producirlo a grandes escalas in vitro, usando diversas técnicas de laboratorio $\left({ }^{1.7}\right)$.

\section{ANTICUERPOS MONOCLONALES E INGENIERÍA GENÉTICA}

La estructura molecular de los AMC puede ser mejorada cambiando, reemplazando o eliminando algunos fragmentos (Tabla 1), lo cual posibilita propiedades terapéuticas más adecuadas.

Una de las variaciones importantes en la estructura de los anticuerpos fue el cambio de las regiones constantes de los AMC de murino a humano, lo cual contribuyó a una menor inmunogenicidad y a una mejor actividad efectora de la región Fc (anticuerpos quiméricos). Un avance mayor se logró cuando se remplazó toda la molécula del anticuerpo con una Ig humana a excepción de los determinantes antigénicos (anticuerpos humanizados) ${ }^{7-11}$ ) (Figura 2).
Tabla 1.- Cambios potenciales en la estructura de las inmunoglobulinas para realzar sus funciones. mejorar su farmacocinética o disminuir su inmunogenicidad.

I. Reducir la respuesta humana antimurina, con la generación de AMC quiméricos o humanizados.

2. Realzar las funciones efectoras de la fracción $\mathrm{Fc}$ (Fragment crystalline).

3. Alterar la farmacocinética del aclaramiento plasmático y a nivel corporal total.

4. Incrementar la penetración del AMC dentro del tejido al cual se dirige.

5. Incrementar la afinidad por ciertos rejidos.

6. Desarrollar inmunoglobulinas conjugadas con drogas, toxinas, modificadores de la respuesta biológica, isótopos, etc., que cumplan funciones terapéuticas especificas.

Recientemente, una alternativa a la producción de AMC por las vías clásicas ha sido el uso de técnicas genéticas moleculares para clonar y expresar genes de anticuerpos, eliminando la fusión celular. Las regiones variables de las inmunoglobulinas son amplificadas a partir de la reacción en cadena de la polimerasa (PCR), para luego ser clonadas dentro de un vector apropiado. El fragmento de anticuerpo resultante puede ser producto secretor de una bacteria o ser expresado en la superficie de la misma o de un fago $\left({ }^{12}\right)$.

\section{ANTICUERPOS MONOCLONALES EN CÁNCER}

Antígenos tumorales. Existen varios tipos de antígenos tumorales, los cuales han permitido generar una amplia variedad de anricuerpos monoclonales; algunos de ellos incluyen: los antígenos oncofetales -como el antígeno carcinoembrionario (CEA)-, la alfa fetoproteína (AFP), la glicoproteína 72 asociada a tumor (TAG 72), antígenos asociados a células en un determinado estadio de diferenciación -como el antígeno común de la leucemia linfoblástica aguda (CALLA) $y$ el antígeno asociado a colon (CAA)-, recepto- 


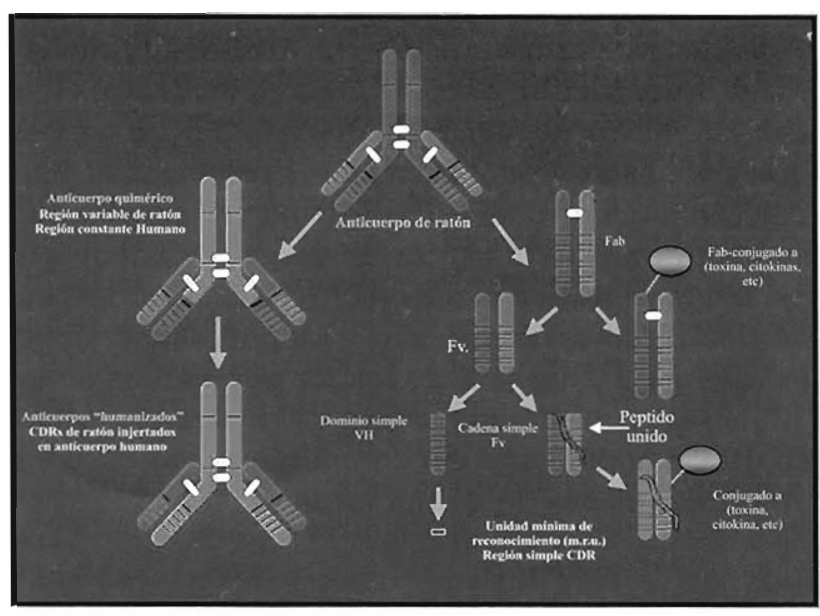

Figura 2.- Evolución de los anticuerpos monoclonales: Fragmentos obtenidos por ingenieria genética.

res de factores de crecimiento y productos de oncogenes, como el factor de crecimiento epidermal, factor de crecimiento derivado de plaquetas, el receptor plasmático Her $2 /$ neu.

Estrategias para el uso de anticuerpos monoclonales. Los AMC pueden ser usados para reconocer segmentos proteicos específicos sobre la superficie celular de las neoplasias; por tanto, ofrecen el porencial enorme de la detección precisa en lesiones recurrentes u oculcas. Los antígenos de la superficie celular pueden ser específicos del tumor o ser expresados en mayor volumen en los tumores que en las células normalmente diferenciadas. Durante décadas han existido diferentes formas de uso de los AMC para el tratamiento del cáncer. Los AMC pueden ser usados solos o conjugados a drogas citotóxicas, citoquinas, radioisótopos o inmunotoxinas, las cuales actuarían selectivamente en el blanco correspondiente (Figura 2). Aún queda por resolver algunos problemas derivados del uso de los AMC conjugados en la terapia del cáncer, entre éstos, asegurar la estabilidad entre el conjugado y la inmunoglobulina, una óptima liberación de i conjugado en el blanco celular, mejorar la penetración de los anticuerpos dentro de los tumores sólidos. A favor debemos decir que los AMC ofrecen una más efectiva localización en las enfermedades microscópicas o metastásicas, mayor afinidad a los antígenos, que otras terapias disponibles.

Los trabajos de investigación y estudios clínicos se desarrollaron casi dos décadas antes de que aparecieran los primeros productos aprobados para su uso dentro del mercado farmacéutico mundial. En el momento aciual, la Food and Drugs Admi. nistration (FDA), en los EE.UU., ha aprobado dos AMC no radiomarcados a finales de 1998, para su uso en enfermedades neoplásicas: rituximab (Mabthera ${ }^{\circledR}$ ) y trastuzumab (Herceptin ${ }^{\circledR}$ ). Asimismo, en Alemania, en el mismo año, se ha aprobado otro AMC, edrecolomab (Panorex ${ }^{\circledR}$ ), en el uso del cáncer colorrectal. En las próximas líneas, por tanto, evaluaremos los puntos más trascendentes de estos productos en la farmacopea anticancerígena actual.

\section{Rituximab}

Es un anticuerpo monoclonal específico para el antigeno CD20, el cual es una fosfoproteína de membrana que regula la conductancia del calcio, siendo expresado en los linfocitos $B$ normales, en los linfomas no Hodgkin derivados de células B en un $93 \%$ y en las células de la leucemia linfocítica crónica hasta en $99 \%\left({ }^{13}\right)$. Este AMC generado a través de la técnica del hibridoma produce citotoxicidad de las células blanco, a través del complemento o citotoxicidad celular dependiente de anticuerpos (Figura 3). Pero, además, rituximab ha demostrado inducir apoptosis in vitro en células B de linfoma ( $\left.{ }^{14, i 5}\right)$. El CD20 no es expresado en células progenitoras de la médula ósea, por lo cual se considera que el tratamiento a este nivel eliminará clonas que expresen el antígeno mencionado, pero no afectarán las células pluripotenciales (stem cell) que posteriormente pueden repoblar la médula ósea con linfocitos normales. Tampoco se expresa en las células plasmáticas y en sus derivados oncogénicos o en componentes de linfocitos $T\left({ }^{13.16}\right)$. Una de las ventajas del blanco antigénico es que no se interna al ligarse al AMC y su expresión es estable $\left({ }^{17}\right)$.

Los estudios realizados para definir la dosificación de rituximab demostraron finalmente que fue efectivo y generalmente bien tolerado a la do- 


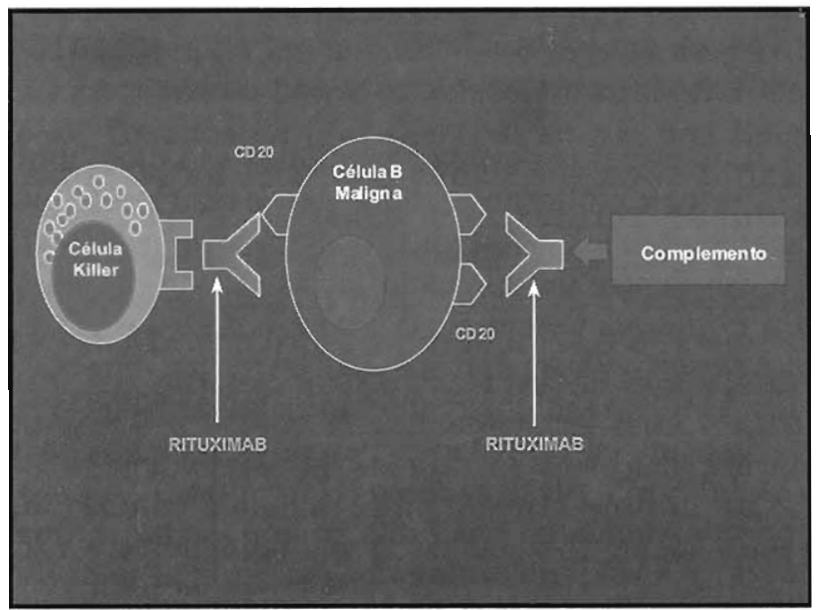

Figura 3.- Reclutamiento de los mecanismos efectores inmunes del huésped a través del dominio Fc humano, en la acción del rituximab.

sis de $375 \mathrm{mg} / \mathrm{m}^{2}$ administrado semanalmente durante 4 ciclos $\left({ }^{18-20}\right)$. El estudio pivote con monoterapia es el 102-05, el cual se realizó en pacientes con linfomas $B$ de bajo grado o folicular (WF: A-D), con enfermedad mensurable, CD20 positivos, resistentes o recurrentes a quimioterapia convencional, en el cual se obtuvo $48 \%$ de respuesta global $(76 / 166)$, con 9 pacientes con respuesta completa $(6 \%)$, bajo el análisis intent to treat (ITT). Sin embargo, $87 \%$ de los pacientes logró algún grado de disminución del tamaño tumoral, sin cumplir con los criterios para respuesta completa o parcial. El tiempo medio de progresión de los 76 que respondieron fue 13,2 meses, con una duración media de respuesta de 11,6 meses. Algunos factores predictivos de respuesta clínica hallados en el estudio se muestran en la Tabla 2; también se observó que del $42 \%$ (70/165) que tuvo rearreglos del gen bcl-2 en sangre periférica al inicio del estudio, $57 \%$ fue indetectable después de la terapia con rituximab y dicho porcentaje aumentó por encima de $70 \%$ un año después $\left({ }^{21}\right)$. Cuando rituximab fue usado como primeta línea (50 pacientes sin tratamiento previo) como monoterapia y en linfomas de grado bajo o foliculares $\mathrm{CD} 20+$, la respuesta global fue $74 \%$ con $28 \%$ de respuestas completas $(6 \%$ no confirmadas) $\left.{ }^{22}\right)$. Un importante aspecto del compor- tamiento farmacocinético del rituximab fue confirmado por el seguimiento en varios estudios realizados por Grillo y col. $\left({ }^{23}\right)$, en el encuentro ASH (American Society of Hematology) del año 1999, quienes observaron que la mejor respuesta al final del tratamiento con rituximab puede conseguirse en los primeros 7 días. Sin embargo, la mayoría de pacientes logrará aquella en el $1^{\circ}$ ó $2^{\circ}$ mes después de finalizado el tratamiento y un porcentaje menor puede incluso llegar a responder por encima de los 10 meses de finalizada la terapia. Esta caracierística difiere totalmente del comportamiento observado en regímenes de quimioterapia.

Tabla 2.- Factores predictivos en el estudio 102-05 $\left({ }^{21}\right)$.

\begin{tabular}{lcc}
\hline Factor predictivo & $\begin{array}{c}\text { Respuesta } \\
\text { global }\end{array}$ & $p<$ \\
\hline WF: B, C ó D & $59 \%$ & 0,01 \\
WF: A & $13 \%$ & \\
No compromiso médula ósea & $61 \%$ & 0,03 \\
Compromiso médula ósea & $42 \%$ & \\
Trasplante previo médula ósea & $78 \%$ & 0,01 \\
Océlulas pluripotenciales & $43 \%$ & - \\
No trasplante previo & & \\
\hline
\end{tabular}

Estudios posteriores han demostrado importantes respuestas, ya sea en retratamiento con el mismo fármaco, en enfermedad masiva, sea con monoterapia o asociado a otros quimioterápicos o citoquinas. El estudio 102-08R evaluó pacientes cuya enfermedad había progresado después de haber respondido a la terapia con rituximab; sesenta pacientes que inicialmente fueron respondedores fueron nuevamente tratados con rituximab con el mismo esquema de tratamiento del estudio $102-05$, lográndose $38 \%$ de respuesta global (ITT). El tiempo medio de progresión sobrepasó los 15 meses y la duración de la respuesta excedió los 16,7 meses $\left({ }^{24}\right)$, lo cual muestra un comportamiento paradójico frente a quimioterapia convencional, en la cual las respuestas globales en pacientes con recaída son más cortas con cada administración nueva, mientras que rituximab aumenta el tiempo libre de progresión y la duración 
de la respuesta en pacientes que previamente respondieron a este AMC. Otro estudio importante a tener en cuenta para el uso de rituximab es su combinación con quimioterapia, con el esquema convencional CHOP. El estudio 102-03 reclutó pacientes con linfomas no Hodgkin de grado bajo o foliculares (WF: A-D), sin enfermedad masiva $(>10 \mathrm{~cm})$ y $\sin$ previo uso de antraciclínicos o antrapirazoles, los cuales recibieron 6 ciclos de CHOP convencional y 6 administraciones de ricuximab ( $\left.375 \mathrm{mg} / \mathrm{m}^{2} / \mathrm{semana}\right)$. De los 40 pacientes que se incluyó, 35 recibieron todas las dosis planificadas y dos pacientes no iniciaron tratamiento (lo cual afecta el análisis ITT). Se logró respuesta completa (RC) en 22 pacientes $(55 \%)$ y 16 respuesta parcial (RP), logrando una respuesta global (RG) del 95\% (ITT, en el cual se considera los pacientes que ingresaron al protocolo, no importando si recibieron o no tratamiento), pero obviamente $100 \%$ de respuesta global en aquellos tratados. La sobrevida media sobrepasa los 40,5 meses y aún no se alcanza $\left({ }^{25}\right)$. Por tanto, el estudio 102-03 demuestra que la combinación rituximab + CHOP es marcadamente más efectiva que cualquiera de los regimenes solos.

Rituximab también ha sido evaluado en linfomas de grado intermedio y alto, tanto en monoterapia como asociado a quimioterapia convencional (CHOP). En dos estudios en linfomas de la zona del manto, los resultados no fueron similares: Foram y col. (27) evaluaron 81 pacientes previamente tratados o sin tratamiento y encontraron $42 \%$ de respuesta global con $15 \%$ de respuestas completas; sin embargo, Ghielmini y col. $\left({ }^{28}\right)$ obtuvieron resultados diferentes en 39 pacientes con el mismo diagnóstico con y sin tratamiento anterior (respuesta global de $22 \%$ sin respuestas completas). Los resultados son aún incipientes para concluir, por lo cual se espera a futuro estudios comparativos que puedan dar resultados más sólidos. Asimismo, se ha realizado estudios clínicos con rituximab en linfomas con WF: D-H, como el estudio 102-09, que evaluó 54 pacientes en dos grupos de tratamiento con rituximab $375 \mathrm{mg} / \mathrm{m}^{2}$ frente a $500 \mathrm{mg} / \mathrm{m}^{2}$, semanalmente por 8 ciclos, sin encontrar diferencia significativa en la respuesta: respuesta global $31 \%$ con $9 \%$ de respuestas completas $\left({ }^{29}\right)$. Otro estudio asociando rituximab a CHOP cada 3 semanas por 6 ciclos (estudio 102-10) en linfomas de grado intermedio y alto (WF: D-H) en 33 pacientes, se obtuvo $97 \%$ de respuesta global (ITT) con $64 \%$ de respuestas completas $\left({ }^{30}\right)$, lo cual demuestra mayor efectividad que la quimioterapia convencional; pero hay que esperar si hay mejoría en la sobrevida y si es menor el número de recurrencias a lo largo del tiempo. Rituximab ha sido probado también en esiudios asociados a transplante de médula ósea autólogo, como agente de purga in vivo, previo a la colección de células progenitoras, en enfermedad mínima residual, con buenos resultados $\left({ }^{31-33}\right)$.

Los efectos adversos recopilados de los diferentes estudios clínicos se los observa en la Tabla 3. Sin embargo, estos efectos disminuyen marcadamente después de la primera infusión (Figura 4). Por tanto, se ha comprobado que rituximab es una poderosa terapia en linfomas no Hodgkin y otras neoplasias que sobreexpresan CD20. Se ha incorporado rápidamente a los regímenes de tratamiento por su excelente eficacia, perfil de seguridad (comparable a otros esquemas) y complementariamente a su mecanismo de acción.

\section{Trastuzumab}

El anticuerpo murino 4D5 ha sido "humanizado" para producir trascuzumab, una inmuno-

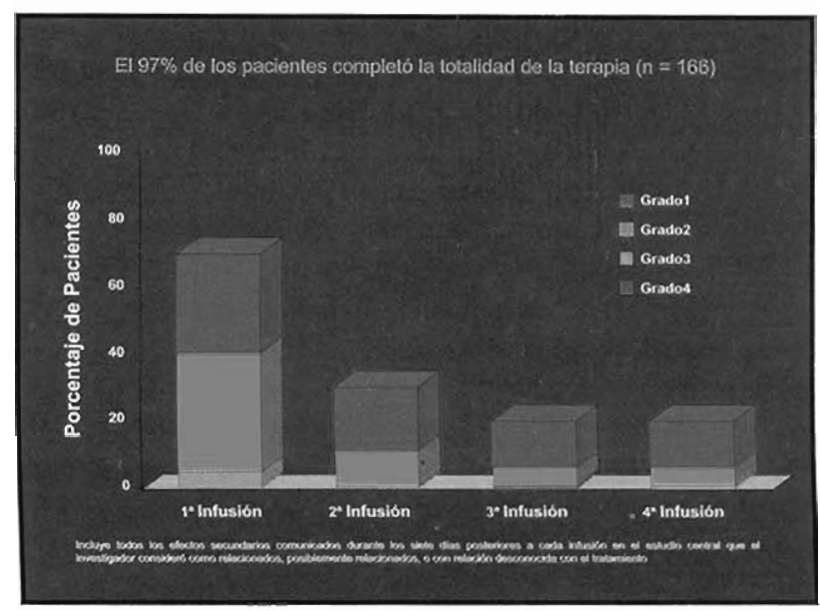

Figura 4.- Efectos secundarios del rituximab, de acuerdo con el período de infusión y la gravedad $\left({ }^{21}\right)$. 
Tabla 3.- Efectos adversos con cl uso de Rituximab $\left.{ }^{(34}\right)$.

\begin{tabular}{|c|c|c|}
\hline & $\begin{array}{l}\text { Incidencia } \\
(\mathrm{n}=354)\end{array}$ & $\%$ \\
\hline Cualquier evento adverso & 336 & 94,9 \\
\hline $\begin{array}{l}\text { Corporal total } \\
\text { Fiebre } \\
\text { Escalofrio } \\
\text { Astenia } \\
\text { Cefalea } \\
\text { Dolor abdominal } \\
\text { Irritacion de la garganta } \\
\text { Dolor }\end{array}$ & $\begin{array}{r}180 \\
115 \\
76 \\
61 \\
38 \\
31 \\
30\end{array}$ & $\begin{array}{r}50,8 \\
32,5 \\
21,5 \\
17,2 \\
10,7 \\
8,8 \\
8,5\end{array}$ \\
\hline $\begin{array}{l}\text { Sistema cardiovascular } \\
\text { Hipotensión } \\
\text { Hipertensión }\end{array}$ & $\begin{array}{l}37 \\
18\end{array}$ & $\begin{array}{r}10,5 \\
5,1\end{array}$ \\
\hline $\begin{array}{l}\text { Sistema digestivo } \\
\text { Náuscas } \\
\text { Vómitos } \\
\text { Diarrea }\end{array}$ & $\begin{array}{l}74 \\
32 \\
27\end{array}$ & $\begin{array}{r}20,9 \\
9,0 \\
7,6\end{array}$ \\
\hline $\begin{array}{l}\text { Sistema sanguíneo y linfático } \\
\text { Leucopenia } \\
\text { Trombocitopenia } \\
\text { Neutropenia } \\
\text { Anemia } \\
\text { Linfadenopatia }\end{array}$ & $\begin{array}{l}41 \\
34 \\
31 \\
23 \\
10\end{array}$ & $\begin{array}{r}11,6 \\
9,6 \\
8,8 \\
6,5 \\
2,8\end{array}$ \\
\hline $\begin{array}{l}\text { Alteraciones metabólicas o } \\
\text { Angioedema } \\
\text { Hiperglicemia } \\
\text { Edema (periférico) }\end{array}$ & $\begin{array}{l}\text { ionales } \\
37 \\
21 \\
20\end{array}$ & $\begin{array}{r}10,5 \\
5,9 \\
5,6\end{array}$ \\
\hline $\begin{array}{l}\text { Sistema musculoesquelético } \\
\text { Mialgia } \\
\text { Artralgia }\end{array}$ & $\begin{array}{l}33 \\
24\end{array}$ & $\begin{array}{l}9,3 \\
6,8\end{array}$ \\
\hline $\begin{array}{l}\text { Sistema nervioso } \\
\text { Vértigo }\end{array}$ & 30 & 8,5 \\
\hline $\begin{array}{l}\text { Sistema respiratorio } \\
\text { Rinitis } \\
\text { Tos en incremento } \\
\text { Broncoespasmo } \\
\text { Sinusitis }\end{array}$ & $\begin{array}{l}37 \\
32 \\
30 \\
20\end{array}$ & $\begin{array}{r}10,5 \\
9,0 \\
8,5 \\
5,6\end{array}$ \\
\hline $\begin{array}{l}\text { Piel y apéndices } \\
\text { Sudor nocturno } \\
\text { Prurito } \\
\text { Rash } \\
\text { Urticaria }\end{array}$ & $\begin{array}{l}47 \\
44 \\
37 \\
28\end{array}$ & $\begin{array}{r}13,3 \\
12,4 \\
10,5 \\
7,9\end{array}$ \\
\hline
\end{tabular}

globulina con secuencias murinas conservadas a nivel de las regiones hipervariables de la fracción Fab, pero el resto de la molécula del anticuerpo es cambiada por técnicas biotecnológicas a componentes humanos. Este $\mathrm{AMC}$ se liga fuertemente al dominio extracelular del receptor HER2. E1 oncogén HER2 codifica para un receptor glicoproteico de membrana, que pertenece a la familia de los receprores de los factores de crecimiento, siendo similar al receptor del factor de crecimiento epidermal ${ }^{35}$ ). En células cancerosas la amplificación del oncogén HER2 resulta en la sobreexpresión celular del receptor correspondiente, pudiendo generar niveles de proteínas entre 10 y 100 veces más altos que su contraparte: las células normales $\left({ }^{36}\right)$ (Figura 5). La sobreexpresión del HER2 puede estar constitutivamente activa, es decir el receptor puede emitir señales sin necesidad del ligando $\left({ }^{37}\right)$; como consecuencia, se activan múltiples vias intracelulares de transducción de señales, que resultan en crecimiento celular no controlado y en la transformación oncogénica consiguiente $\left({ }^{38.30}\right)$. El receptor HER2 es sobreexpresado en aproximadamente $25 \%$ a $30 \%$ de tumores cancerosos mamarios $\left({ }^{40.41}\right.$ ) y casi siempre en cáncer inflamatorio de la mama $\left({ }^{42}\right)$. Las pacientes que sobreexpresan HER2 han mostrado un tiempo de sobrevida global menor y una mayor tasa de

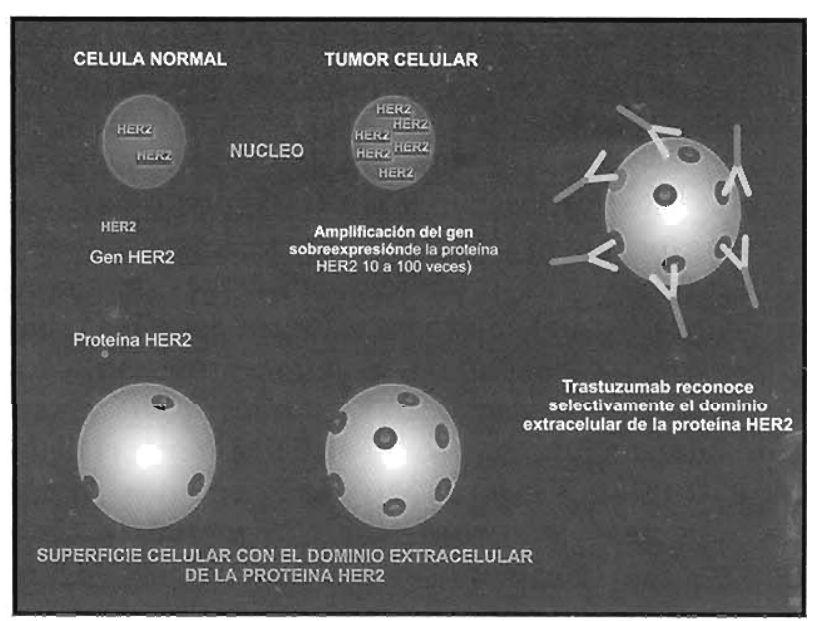

Fig.ura 5.- Amplificación del gen HER2, con la consiguiente sobreexpresión del receptor de supcrficie y la acción de trastuzumab. 
recurrencia cumoral $\left({ }^{40.43}\right)$, sin embargo no todos los escudios han demostrado esta asociación, sobre todo en pacientes con cáncer de mama con ganglios linfáticos negativos $\left({ }^{11}\right)$, en contraposición a otros estudios que, utilizando análisis univariables, encontraron que la presencia de HER2 es un factor pronóstico adverso, pero no fue confirmado con el uso de análisis multivariables $\left({ }^{44-49}\right)$. Asimismo, existen estudios que han correlacionado la sohreexpresión de HER2 como factor predictivo de respuesta a terapia. con pacientes con tumores que expresan receptores estrogénicos posicivos (RE+) y que fucron tratados con tamoxifeno $\left({ }^{50.5 b}\right)$, aquellos que fueron tratados con CMF ( ${ }^{44.46}$ ) o con esquemas basados en antraciclínas $\left({ }^{52}\right)$, pero cuyos resultados no mostraron significación estadística. a pesar que existió una tendencia evidence que posiblemence sea confirmada en estudios posteriores, con diseños prospectivos, aleatorizados y con suficience muestreo para definir el papel predicrivo y pronóstico de la sobreexpresión del HER2 y que permita tratamientos más agresivos y dirigidos a grupos de riesgo.

A nivel sérico se ha derectado rambién la fracción extracelular del receptor HER2, el cual sería causado por una enzima proteolitica (53) y otros consideran que podría suceder a nivel del ARNm por razones no entendidas $\left({ }^{54.55}\right)$ : sin embargo, su presencia ha sido asociada con falta de respuesta a hormonoterapia en cáncer avanzado, acortamiento del tiempo libre de enfermedad y de la sobrevida global $\left({ }^{56,57}\right)$, respuesta a adriamicina $\left({ }^{58}\right)$, metásrasis a distancia, falta de expresión de receptores hormonales y pobre pronóstico $\left({ }^{5 /}\right)$.

Los estudios farmacológicos preclínicos indicaron que el AMC anti-HER2 trastuzumab posee potente actividad antitumoral $\left(^{00.61}\right)$, ejerciendo su mecanismo de acción a través varios mecanismos. Puede antagonizar la función generadora de señales de rransducción, impidiendo finalmente el crecimiento e indiferenciación tumoral. Sin embargo, considerando los antecedentes antes mencionados, con respecto al HER2 como factores predictivos y pronósticos aún no bien determinados, los diferentes estudios con trastuzumab han sido llevados a cabo en pacientes con cáncer de mana metastásico con sobreexpresión del HER2.
Los estudios de fase 1 , realizados básicamente para evaluar la seguridad de la medicación. señalaron la vía endovenosa como la más ióónea. asi como la administración semanal. de acuerdo a las características farmacocinéricas. Trastuzumab tue bien tolerado con dosis semanales reperidas por via endovenosa, en un rango de dosis de 10 a $500 \mathrm{mg}\left({ }^{62}\right)$. Tomando en cuenta los hallazgos en la fase I, se realizó dos estudios de tase II, en los cuales se inició con una dosificación inicial alta (250 mg) y un manrenimiento con $100 \mathrm{mg} / \mathrm{sema-}$ na, observándose una aceptable actividad en cáncer melastásico de mama y buena colerancia $\left({ }^{63.64}\right)$. Para establecer claramente los beneficios y riesgos del trastuzumab en cáncer de mama metastásico, como monoterapia o en combinación con quimioterapia, se realizó un conjunto pivote de estudios de fase III. El primero de ellos fue un estudio abierto, aleatorizado comparativo de trastuzumab con el regimen AC (antraciclínico + ciclofosfamida) o paclitaxel (P), contra quimioterapia sola ( $A C$ O $P$ ) en cáncer de mama mecastásico HER2 + (estudio H0648g); la razón de elegir AC en el estudio fue por ser el régimen de primera línea en estos casos, y su uso con trastuzumab fue evaluado en los estudios preclínicos (Baselga y col.) $\left({ }^{60}\right)$; y en el caso de haber sido tratados previamente con antraciclínicos, se les daba la oportunidad de recibir uno de los brazos con paclitaxel (Figura 5). Para evaluar más objecivamente las respuestas a lá terapia se creó un comité que trabajó ciegamente los casos. Los resultados preliminares de este estudio fueron presentados en el Congreso Americano de Oncología Clínica (ASCO) en 1998 $\left({ }^{(35}\right)$. Nuevos resultados de este escudio fueron presentados en el ASCO DE 1999, por Norton y col. $\left({ }^{65}\right)$, con un seguimiento promedio de 29 meses (rango 21-42), observándose que la sobrevida global media fue significativamente mejor en el grupo de trastuzumab + quimioterapia (QT) que aquellos que recibieron sólo QT $(25,4$ frente a 20,3 meses; $p=0,025$ ) en $25 \%$; estos resultados son aún más convincentes, ya que a partir del $5^{\circ}$ mes se inició tratamiento adicional con los pacientes refraciarios a $Q T$, habiéndose incluido dentro de estos complementos terapéuticos a trastuzumab hasta en $65 \%$ de este grupo.

Para evaluar la seguridad y eficacia de trastuzumab como agente único, se realizó un se- 
gundo estudio $(\mathrm{H} 0649 \mathrm{~g})$ en pacientes con cáncer de mama metastásico HER2 +, que habían recurrido en su enfermedad después de uno o dos ciclos de quimioterapia previa; la dosificación de trastuzumab fue similar al estudio anterior y se incluyó 222 pacientes, obteniéndose una respuesta global de $15 \%$ (IC95\%: $10 \%-20 \%$ ), con $4 \%$ de respuestas completas; pero además se obtuvo $7 \%$ de respuestas menores y $30 \%$ de estabilización de la enfermedad, la duración media de la respuesta fue 8,4 meses y el estimado Kaplan-Meier de la sobrevida media fue 13 meses $\left({ }^{67}\right)$. En los últimos congresos mundiales de oncología médica se ha presentado algunos resúmenes interesantes sobre trastuzumab asociado a paclitaxel $\left({ }^{68}\right)$, docetaxel $\left({ }^{69}\right)$, cisplatino $\left({ }^{70}\right)$, los cuales demuestran la efectividad de trastuzumab asociada a QT. También se ha reportado estudios considerando la calidad de vida de los pacientes $\left({ }^{71}\right)$. Así Lieberman y col. $\left({ }^{72}\right)$ presentaron en el ASCO 1999 la valoración del efecto de la terapia anti-HER2, como agente único en la calidad de vida de los pacientes que ingresaron al estudio de Cobleigh y col. $\left({ }^{67}\right)$, demostrando una significativa mejoría en el funcionamiento social y global, sin desmedro de otros aspectos, a las 48 semanas del tratamiento, tanto en el grupo que respondió como en el que no respondió a la terapia. Si se considera solamente el grupo respondedor, la calidad de vida mejora notablemente y en todos los aspectos. Baselga y col. $\left({ }^{73}\right)$, presentaron en el último encuentro europeo de oncología clínica (ECCO 99) el estudio sobre la calidad de vida en las pacientes que ingresaron a la fase III de trastuzumab con QT $\left(^{65}\right)$, concluyendo que las pacientes que recibieron trastuzumab mostraron tendencias de una mejoría en los objetivos primarios y secundarios de calidad de vida de acuerdo al cuestionario QLQ C-30 $\left({ }^{74}\right)$ aplicado.

Los efectos adversos de trastuzumab pueden resumirse en la Tabla 4, derivado del estudio de fase III de Herceptin con QT $\left({ }^{65}\right)$. Es importante considerar dentro de estos la toxicidad a nivel cardiaco, la cual tiene diversas explicaciones en relación a HER2 y 3 , los cuales son expresados a nivel miocárdico fetal en el feto y adulto respectivamente $\left({ }^{75}\right)$. La asociación de trastuzumab con la combinación AC fue más cardiotóxica que con paclitaxel, lo que nos permite inferir que Herceptin potencia el efecto tóxico de la adriamicina a nivel cardiaco. Sin embargo, el $80 \%$ de los pacientes mejoran la disfunción cardiaca con tratamiento estándar y en el estudio irastuzumab fue discontinuado en el $8 \%$ de los pacientes que recibieron además $\mathrm{AC}\left({ }^{65}\right)$.

\section{Edrecolomab}

Es un $\mathrm{AMC}$ murino $\operatorname{lgG}_{2, \mathrm{~s}}$ que reconoce como antígeno una proteína con 37-40 kd encontrada en varios adenocarcinomas y en tejido epitelial normal $\left({ }^{76}\right)$, la cual posiblemente esté relacionada con la adhesión celular. Debido a su origen, este AMC puede producir anticuerpos anti-IgG $\mathrm{Ig}_{2 \mathrm{i}}$ murinos $\left(\mathrm{ab}_{2}\right)$ hasta en 50 a $100 \%$ de pacientes $\left({ }^{77.78}\right)$. Sin embargo, esta reacción, lejos de disminuir su actividad por modulación inmune, puede llegar a ser importante en la respuesta $\left({ }^{79}\right)$, pues edrecolomab parece evocar actividad antitumoral indirectamente por inducción de una respuesta al anticuerpo de tipo antidiotípica, formando anticuerpos antiantiidiotípicos $\left(a b_{3}\right)$. los cuales reconocen como blanco el mismo antígeno que edrecolomab. Se ha reportado que los pacientes que más forman estos anticuerpos tienen ventajas de respuesta frente a aquellos que forman pequeñas cantidades o no forman $\mathrm{ab}_{3}\left({ }^{80.81}\right)$. Edrecolomab posiblemente destruye células que contienen el antígeno respectivo a través de mecanismos citotóxicos endógenos que incluyen citotoxicidad mediada por células dependiente de anticuerpos y complemento $\left.{ }^{82}\right)$.

Las respuestas en los diversos estudios en adenocarcinomas metastásicos de colon, no fueron satisfactorias $\left({ }^{77.83-86}\right)$. Sin embargo, el estudio realizado por Riethmuller y col. $\left({ }^{87}\right)$ con edrecolomab, como tratamiento adyuvante, en pacientes con estadio $C$ de Dukes, a los cuales se les realizó resección completa de la tumoración y fueron posteriormente aleatorizados para observación (90 pacientes) o para un régimen posoperatorio con edrecolomab $500 \mathrm{mg} \mathrm{EV}$ de inicio y $100 \mathrm{mg}$ EV una vez al mes por 4 meses $(99$ pacientes), después de 7 años de seguimiento existió una diferencia estadistica significativa en ambos grupos en la curva de sobrevida global y en el tiempo promedio de recurrencia del tumor (32\% y $23 \%$ mayor en el grupo con edrecolomab), similares a los hallados con el régimen 5FU y 
Tabla 4.- Efectos adversos con el uso de Trastuzumab ( $\left.{ }^{24}\right)$.

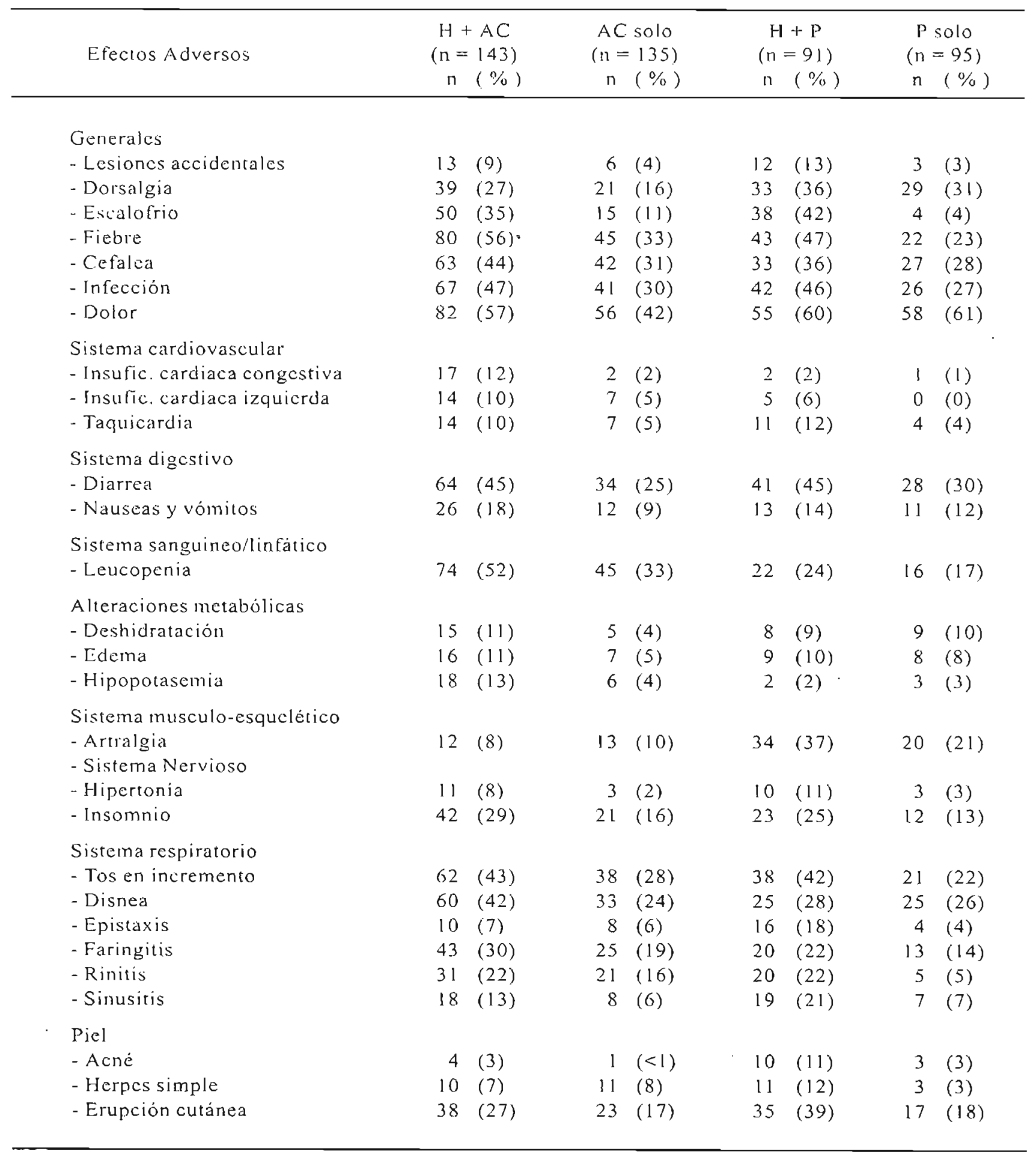

H: Herceprin AC: Anmaciclina + ciclofosfamida P: Paclitarel 
leucovorina. Se espera resultados de los estudios comparativos emprendidos con terapia combinada con agentes antineoplásicos con o sin edrecolomab. Se ha realizado también estudios de edrecolomab en cáncer de colon avanzado, con otros agentes, como factor estimulante de colonias de granulocitos y monocitos (GM-CSF) ${ }^{88}$ ), regímenes de QT con $5 \mathrm{FU}\left({ }^{89}\right)$, interferón $\mathrm{g}\left({ }^{90.91}\right)$ con resultados poco alentadores. Edrecolomab también ha sido estudiado en otros tumores, como cáncer de páncreas y cáncer de mama avanzado, con respuestas no relevantes $\left({ }^{92-94}\right)$.

Los efectos colaterales del edrecolomab son bastante similares a los otros dos anticuerpos monoclonales presentados en esta revisión. Asimismo, ellos se presentan con mayor intensidad y frecuencia durante el primer ciclo de tratamiento. Posiblemente haya más riesgo de choque anafiláctico, tratándose de un anticuerpo murino. En un estudio como adyuvante en cáncer de colon, Schmoll y col. $\left({ }^{95}\right)$ publicaron los efectos adversos de edrecolomab en 135 pacientes: diarrea $30,7 \%$, náuseas $20,2 \%$, dolor abdominal $18,7 \%$, eritema o bochornos $8,3 \%$, vómitos $7,9 \%$ y reacciones anafilácticas $2,9 \%$. Del total, 32 pacientes desarrollaron eventos adversos de grado III o IV valorados con la propuesta de la OMS, por lo cual discontinuaron el estudio. Por tanto, edrecolomab está posicionado básicamente en el cáncer de colon como tratamiento adyuvante postoperatorio en pacientes con un estadio $C$ de Dukes.

\section{ANTICUERPOS MONOCLONALES: EL FUTURO}

Desde su descubrimiento, los AMC han evolucionado a una velocidad vertiginosa, habiéndose logrado mejorar todas sus características y su adaptación para servir tanto en el área diagnóstica como terapéutica. Sin embargo, algunos problemas han surgido en áreas clínicas particulares que están siendo resueltas paulatinamente, lográndose cada vez mejores posibilidades de eficiencia y menor toxicidad asociada. Uno de los problemas más importantes fue la generación de anticuerpos contra las inmunoglobulinas murinas, lo cual ha sido casi eliminado con la creación de los anticuerpos monoclonales humanizados, en los cuales se conserva solamente las regiones hipervariables murinas, mientras el resto de la molécula ha sido reemplazado por componentes humanos. Actualmente, ya se ha comenzado a experimentar la formación de AMC fuera de componentes murinos y a partir de material genético, con lo cual a futuro obtendríamos mayor pureza, afinidad y selectividad. Por tanto, el uso de AMC en el fururo evolucionará acorde a las necesidades del hombre y a su creatividad.

\section{BIBLIOGRAFÍA}

1) Abbas AK, Lichtman AH, Pober JS. Antibodies and antigens. En: Cellular and Molecular Immunology, $2^{\text {id }}$ ed. Philadelphia:W.B. Saunders Company. 1994:33-64.

2) Rojas W. Inmunidad hunoral - Linfocito B. En: Inmunologia, $10^{\mathrm{ma}}$ ed. Colombia:Corporación para la Investigación Biológica. 1995:92-110

3) Carayannopoulos L, Capra D. Inmunoglobulins: Structure and function. En: Paul WE. Fundamental Inmunology, $3^{\text {rd }}$ ed. Néw York: Raven Press. 1993:283-314.

4) Alzari PM, Lascombe M, Poljak RJ. Three-dimensional siruclure of antibodies. Ann Rev Immunol 1988;6:555-80.

5) Tonegawa $S$. Somatic generation of antibody diversity. Nature 1983:302:575-81.

6) Köhler G, Milstein C. Continuous cultures of fused cells secreting antibody of predefined specificity. Nature 1975;256:495-97.

7) Maloney DG, Levy R, Campbell MJ. Monoclonal antibody therapy. En: Mendelsohn J, Howley PM, Israel MA, Liolta LA. The Molecular Basis of Cancer, Philadelphia:W.B. Saunders Company, 1995:460-510.

8) Hosono M, Endo K, Sakahara H, et al. Human/mouse chimeric antibodies show low reacsivity with human antimurine antibodies (HAMA). Br J Cancer 1992;65;197-203.

9) Shaw DR, Khazaeli MB, LoBuglio AF. Mouse/human chimeric antibodies 10 a tumour-associated antigen: Biologic activity of the four human IgG subclasses. I Nall Cancer Inst 1988;80: 1553-58

10) Co MS, Queen C: Humanised antibodies for therapy. Nature 1991;351:501-13.

11) Chaudhary VK, Queen C Junghans RP, et al. A recombinant immunotoxin consisting of two antibody $V_{2}$ riable domains fused to Pseudomonas exotuxin. Nature 1989;339:394-99.

12) Huse WD, Sastry L, Iverson SA, et al. Generation of a large combinatorial library of the inmunoglobulin repertoire en phage lambda. Science 1989;246:1275-82.

13) Anderson KC, Bates MP, Slaughenhoupt BL, et al. Expression of human B cell-associaled antigens on leukenvias and lymphomas: a model of human B cell differentiation. Blood 1984:63:1424-33. 
14) Reff ME, Camer K, Chambers KS, et al. Depletion of B cells in rivo by a chimeric mouse human monoclonal antibudy 10 CD20. Blood 1994:83:435-45.

15) Mathas S, Kommert K, Dörken B, et al. Anti-C.D20 antibody mediated apoptosis is dependent on caspase 3 activation [Abstract 1671]. Blood 1998;92:405a.

16) Nadler LM, Rilz J. Hardy R, et al. A unique cell surface antigen identifying lymphoid malignancies of $\mathrm{B}$ cells origin. J Clin Inves 1981:67:134-40.

17) Maloney DG, Smith B, Appelbaum FR. The anti-tumor effect of monoclonal anti-CD20 antibody (mAb) therapy includes direct anri-proliferative activity and induction of apoptosis in CD20 positive non-Hodgkin's lymphoma (NHL) cell lines [Abstract 2537]. Blood 1996;88-637a.

18) Maloney DG, Liles TM, Czerwinsky DK, et al. Phase I Clinical trial using escalating single-dose infusion of chimeric anti-CD20 monoclonal antibody (IDEC-C2B8) in patients with recurrent B-cell lymphoma. Blood 1994;84:2457-66.

19) Maloney DG, Grillo-López AJ, Bodkin DJ, et al. IDECC2B8: results of a phase I multiple-dose trial in patients with relapsed non-Hodgkin's lymphoma. J Clin Oncol 1997; 15:3266-74.

20) Maloney DG, Grillo-López A J, White CA, et al. IDECC2B8 (Rituximab) anti-CD20 monoclonal antibody therapy in patients with relapsed low grade non-Hodgkin's lymphoma. Blood 1997;90:2188-95.

21) McLaughin P, Grillo-López AJ, Link BK, et al. Rituximab chimeric anti-CD20 monoclonal antibody therapy for relapsed indolent lymphoma: half of patients respond to a four dose treatment program. J Clin Oncol 1998; 16:2825-33.

22) Solal-Celigny et al. Rituximab as firtst line treatment of patient with follicular lymphoma (FL) and low burden tumor: Clinical and molecular evaluation. [Abstrace 2802]. $41^{\text {st }}$ annual meeting of the American Society of Hematology 1999.

23) Grilio-López et al. Responders to Riruximab show continued cumor regression over time and a progression free survival that correlates with response classification. [Abstract 390]. $4 \mathrm{~L}^{\mathrm{st}}$ annual meeting of the American Society of Hematology 1999.

24) Davis T, Levy R, White CA, et al. Rituximab: Phase II (PIl) retreatment $(\mathrm{R} r \mathrm{Rx})$ study in patients (pts) with lowgrade or follicular (LG/F) NHL |Absiract 1710]. Blond 1998;92:(10 Suppl 1):414a.

25) Czuczman MS, Grillo-López AJ, White CA, et al. Treatment of patients with low-grade B-cell lymphoma with the combination of chimeric anti-CD20 monoclonal antibody and CHOP chemotherapy. J Clin Oncol 1999; 17:268-76.

26) Czuczman et al. Rituxinab/CHOP chemoimmunotherapy in parients with low grade lymploma (LG/F NHL): Free survival afler three years (median) follow-up. [Abstract 432]. $41^{\text {st }}$ annual meeting of the American Sociely of Hematology 1999 .

27) Foran JM. Cunningham D, Coiffier B, ef al. Immunotherapy of mantle cell lymphoma/ MC.L with
Riruximab (Rmab): duration of response \& analysis of factors associated with response [Abstract 101]. Ann Oncol 1999;10 (Suppl 3):33.

28) Ghielmini M, Hsu-Schmitz SF, Burky K, et al. The effect of Rituximab on patients with follicular and mantle cell lymphoma [Abstrac! 102]. A.nn Oncol 1999;10 (Suppl 3):33.

29) Coiffier B, Haioun C, Ketterer N, et al. Rituximab (antiCD20 monoclonal antibody) for the treatment of patients with relapsing or refractory aggressive lymphoma: a multicenter phase II study. Blood 1998;92:1927-32.

30) Vose JM, Link BK, Grossbard MI, et al. Phase II study of Rituximab in combination with CHOP chemotherapy in patients with previously untreated intermediate or high-grade non-Hodgkin's lymphoma (NHL) [Abstract 195]. Ann Oncol 1999: 10(Suppl 3):58.

31) Brugger W. Manz M, Grunebach F, et al. Anti-CD20 Rituximab treatment after high-dose therapy and CD34+ blood stem cell transplant for newly diagnosed follicular and mantle cell non-Hodgkin's lymphoma [Abstract 622]. Ann Oncol 1999: 10(Suppl 3): 1.69.

32) Flinn IW, O'Donnell P, Noga SJ, et al. In vivo purging and adjuvant immunotherapy with Rituximab during PBSC transplant for NHL [Abstract 259]. Ann Oñco] 1999:10(Suppl 3):75

33) Buckstein R, Imrie K, Spaner D, et al. In vivo purging with Rituxan: effects in stem cell mobilization efficacy, harvest purity, cytokine profile and engraftment. in patients with relapsed follicular lymphoma undergoing autologous [Abstract 613]. Ann Oncol 1999;10(Suppl 3): 167.

34) Data on file. Laboratorios Roche.

35) Perry CM, Wiseman LR.Trastuzumab. Biodrugs 1999; 12:129-35.

36) Venter DJ, Tuzi NL, Kumar S, et al. Overexpression of the c-erbB-2 oncoprotein in human breast carcinomas: Immuno-histological assessment correlates with gene amplification. Lancet 1987;2:69-72.

37) Lonardo F, Dí Marco E, King CR, et al. The normal erbB-2 product is an atypical receptor-like tyrosine kinase with constitutive activity in thje absence of ligand. New Biol 1990;2:992-1003.

38) Bishop JM. Molecular themes on oncogenesis. Cell 1991:64:235-48.

39) Bishop JM. Viral oncogenes, Cell 1985;42:23-38.

40) Slamon DJ, Clark GM, Wong SG, et al. Human breası cancer: Correlation of relapse and survival with amplification of the HER-2/neu oncogene. Science 1987;235:177-82.

41) Slamon DJ, Godolphin W, Jones LA, et al. Studies of the HER-2/neu protooncogene in human breast and ovarian cancer. Science 1989;244:707-12.

42) Charpin C, Bonnier P, Khouzami A, et al. Inflammatory breast carcinoma: an immunohistochemical study using monoclonal anti-HER-2/neu, pS2, cathepsinj, ER and PR. Anticancer Res 1992;12:591-8.

43) Seshadri R, Firgaira FA, Horsfall DJ, et al, Clínical significance of $\mathrm{HE}-2 /$ neu oncogene amplification in primary breasi cancer. J Clin Oncol 1993;11:1936-42. 
44) Allred DC, Clark GM, Tandon AK, et al. HER-2/neu in node-negative breast cancer: Prognostic significance of overexpression influenced by the presence of in situ carcionoma. J Clin Oncol 1992; 10:599-605.

45) Yuan J, Hennessy C, Givan AL, et al. Predicting outcome for patients with node negative breast cancer: $A$ comparative study of the value of how citometry and cell image analysis for determination of DNA ploidy. $\mathrm{Br} \mathrm{J}$ Cancer 1992;65:461-5.

46) Gusterson BA, Gelber RD, Goldhirsch S, et al. Prognostic importance of c-erbB-2 expression in breast cancer. International (Ludwig) Breast Cancer Study Group. J Clin Oncol 1992;10:1049-56.

47) Bianchi S, Paglierani M, Zampi G, et al. Prognostic significance of $\mathrm{c}$-erbB-2 expression in node negative breast cancer. Br J Cancer 1993;67:625-9.

48) Noguchi M, Koyasaki N, Ohta N, et al. C-erbB-2 oncoprotein expression versus internal mammary lymph node metastases as additional prognostic factors in patiens with axillary lymph node-positive breast cancer. Cancer 1992;69:2953-60.

49) Clabsen PC, van de Velde CJH, Duval C, et al. P53 protein accumulation and response to adjuvant chemotherapy in premenopausal women with node negative breast cancer. J Clin Oncol 1998;16:470-9.

50) Elledge RM, Green S, Ciocca D, et al. HER-2 expression and response to camoxifen in estrogen receptor-breast cancer: a Southwest Oncology Group Study. Clin Cancer Res 1998;4:7-12.

51) Carlomagno C, Perrone F, Gallo C, et al. C-erbB-2 overexpression decreases the benefit of adjuvant tamoxifen in early-stage breast cancer without axillary lymph node metastasis. J Clin Oncol 1996; 14:2702-8.

52) Paik S, Bryant J, Park C, et al. ErbB-2 and response to doxorubicin in patients with axillary lymph node-positive. hormone receptor-negative breast cancer. J Natl Cancer Inst 1998;90:1361-70.

53) Pupa SM, Ménard S, Morelli D, Pozzi B, De Palo G, Colnaghi MI. The extracellular domain of the c-erbB-2 oncoprotein is released from tumor cells by proteolytic cleavage. Oncogene 1993;8:2917-23.

54) Scott GK, Robles R, Park JW, et al. A truncated intracellular HER2/neu receptor produced by alternative RNA processing affects growth of human carcinoma cells. Mol Cell Biol 1993:13:2247-57.

55) Gebhardt F, Zänker KS, Brandt B. Differential expression of alternatively spliced c-erbB-2 mRNA in primary tumors, lymph node metastases, and bone marrow micrometastases from breast cancer patients. Biochem Biophys Res Commun 1998;247:319-23.

56) Leitzel K, Teramoto Y, Konrad K, et al. Elevated serum c-erbB-2 antigen levels and decreased response to hormone therapy of breast cancer. J Clin Oncol 1995:13:1129-35.

57) Yamauchi H, O'Neill A, Gelman R, et al. Prediction of response to antiestrogen therapy in advanced breast cancer patients by pretreatment circulating levels of extracellular domain of the HER-2/c-neu protein. J Clin Oncol 1997; 15:2518-25.

58) Yang L, Liocheva V, Colvin OM, Harris LN. Different response to adriamycin vs Cytoxan-based chemotherapy in ErbB2 positive breast cancer is associated with alteration in topoisomerasa IIa (topo IIa) expression [Abstract]. Breast Cancer Res Treat 1998;50:231.

59) Molina R, Jo J, Filella X, et al. C-erbB-2 oncoprotein, CEA and CA 15.3 in patiens with breast cancer: prognostic value. Breast Cancer Res Treat 1998:51:109-19.

60) Baselga J, Norton L, Albanell J, et al. Recombinant humanized anti-HER2 antibody (Herceptin) enhances the antirumor activity of paclitaxel and doxorubicin against HER2/neu overexpression human breast cancer xenografts. Cancer Res 1998; 582825-31

61) Pietras RJ, Pegram MD, Finn RS, et al. Remission of human breast cancer xenografts on therapy with humanized monoclonal antibody so HER-2 receptor and DNA-reactive drugs. Oncogene 1998; 17:2235-49.

62) Shak S. Overview of the trastuzumab (Herceptin) anti-HER2 monoclonal antibody clinical program in HER2overexpressing metastatic breast cancer. Semin Oncol 1999:26(Suppl 12):71-7.

63) Baselga J, Tripathy D, Mendelsobn J, et al. Phase II study of weekly intravenous recombirant humanized antipI 85 HER2 monoclonal antibody in patients with HER2/ neu overexpressing metastatic breast cancer. J Clin Oncol 1996; 14:737-44.

64) Pegram MD, Lipton A, Hayes DF, et al. Phase II siudy of receptor-enhanced chemosensitivity using recombinant humanized anti-p185 HER2/neu monoclonal antibody plus cisplatin in patients with HER2/neu-overexpression metastatic breast cancer refractory to chemotherapy treatment. J Clin Oncol 1998;8:2659-71.

65) Slamon D, Leyland-Jones B, Shak S, et al. Addition of Herceptin (humanized anti-HER2 antibody) to first line chemotherapy for HER2 overexpressing metastatic breast cancer (HER2 +/MBC) markedly increases anticancer activity: A randomized, multinational controlled phase Ill tria) [Abstract). Proc Am Soc Clin Oncol 1998:17:98a.

66) Norton et al. Overall survival advantage to simultaneous chemotherapy plus the humanizes anti-HER2 monocional antibody herceptin in HER2 overexpressing (HER2+) metastatic breast cancer. [Abstract 483]. 35th annual meeting of the American Society of Clinical Oncology 1999.

67) Cobleigh MA, Vogel CL, Tripathy D, et al. Efficacy and safety of Herceptin (humanized anti-HER2 antibody) as a single agent in 222 women with HER2 overexpression who relapsed following chemotherapy for metastatic breast cancer [Abstract]. Proc Am Coc Clin Oncol 1998; 17:97a.

68) Fornier M, et al. Weekly herceptin + 1 hour taxol: Phase II study in HER2 overexpressing (HER2 +) and nonoverexpressing (HER2-) metastatic breast cancer. [Abstract 482]. $35^{\text {th }}$ annual meeting of the American Society of Clinical Oncology 1999. 
69) Burris HA, Hainesworth JD, Albain K. Huntington $\mathbf{M}$, et al. Phase II trial of docelaxel and Herceptin as first-or second- line clyemotherapy for women with metastatic breast cancer whose tumours overexpress HER2. ECCO 99.

70) Pegram M. Slamon D. Herceprin ${ }^{\circledast}$ plus cisplatin is acrive in patients with metastatic breast cancer. ECCO 99.

71) Osolsa D, Burchmore M. Health-related quality of life in women with metastatic breast cancer treated with Irastuzumab (Herceptin). Semin Oncol 1999:26(Suppl 12):84-88.

72) Lieberman [Abstract 16613]. Health-related quality of life in metastatic breast cancer patients who responded 10 antiHER2 therapy over 48 weeks. ECCO 99

73) Baselga J, Kerrigan M, Burchmore M. Asli M. Healthrelated quality of life (HRQL) in women with HER2overexpressing melastatic breast cancer $(\mathrm{MBC})$ in a phase III study of Herceptin plus chemotherapy versus chemotherapy alone. ECCO 99

74) Aaronson NK, Ahmedzai S, Bergman B, et al. The european organization for research and treatment of cancer QLQ-C30: A quality-of-life instrument for use in international clinical trials in oncology. J Natl Cancer Inst 1993:85:365-76.

75) Ewer MS, Gibbs HR, Swafford J, Benjamin RS. Cardioloxicity in patients receiving trastuzumab (Herceptin): primary coxicity, sinergistic or sequential stress, or surveillance artifact? Semin Oncol 1999;26(Suppl 12):96101

76) Gottlinger HG, Funke I, Hohnson JP, et al. The epithelial cell surface antigen PANOREX, a target for antibodymediated tunor therapy: Its biochemicill nature. tissue distribution and recognition by different monoclonal anibodies. In J Cancer 1986:38:47-53.

77) Sears HF, Mattis J, Herlyn D, et al. Phase-1 clinical urials of monoclonal antibody in treasment of gastrointesinal lumors. Lancel 1982;1:762-65.

78) Blottière HM, Steplewski Z, Herlyn D, at al. Human antimurine imnunoglobulin responses and immune functions in cancer patients receiving murine monoclonal antibody therapy. Hum Antibodies Hybridomas 1991:2:16-25.

79) Herlyn D, Harris D, Azloudik J, et al. Immunomodulatory activity of monoclonal anti-idiotypic antibody 10 anticolorectal carcinoma antibody $\mathrm{CO} 17-1 \mathrm{~A}$ in animals and parients. J limmunother 1994:15:303-11.

80) Fagerbert J, Ragnhamnar P, Liljefors M, et al. Humoral anti-idiotypic and anti-anti-idiotypic immunc response in cancer parients treated with nonoclonal ancibody 17-1 A. Cancer Immunol Immunother 1996:42:81-7

81) Frödin JE, Faxas ME, Hagström B, et al. Induction of anti-idiotypic $\left(a b_{2}\right)$ and anti-anti-idiotypic $\left(a b_{3}\right)$ antibodies in patients treated with the mouse monoclonal antibody 17 I A ( $a b_{1}$ ). Relation to the clinical outcome -an important antitumoral effector function? Hybridoma 1991:10:421-31.

82) Holz E, Gruber R, Riethmüller G, et al. Monoclonal antibodies in cancer therapy: new perspectives after the colorectal carcinoma trial. Clin Immunother 1996:5:2 14-22.

83) Shen JW, Atkioson B, Koprowski H, et al. Binding uf nurine immunoglobulin 10 human lissues atier insmunotherapy with anticolorectal carcinoma nonoclunat antibody. Int J Cancer 1984:33:465-68.

84) Sears HF, Herlyn D, Steplewski Z, et al. Effects of monoclonal antibody immunotherapy on parients with gastrointestinal adenocarcinoma. J Biol Response Modif 1984;: :138-50.

85) Sears HF, Herlyn D, Steplewski Z, et al. Phase Il clinical trial of a murine monoclonal antibody cytotoxic for gastrointestinal adenocarcinona. Cancer Res 1985:45:591013.

86) LoBuglio AF, Saleh MN, Lee J, et al. Phase [ trial of multiple large doses of murine monoclonal antibody $\mathrm{CO} 17$ 1A. J Natl Cancer Inst 1988:17:932-36.

87) Riethmuller G, Holz E, Schlimok G, et al. Monocional ancibody therapy for resected Dukes $C$ colorectal cancer: Seven-year outcome of a multicenter randomized trial. J Clin Oncol 1998:1788-94.

88) Ragnhammar P, Fagerber J, Frödin JE, et al. Elfect of monoclonal antibody 17-1A and GM-CSF in patients with advanced colorectal carcinoma: long lasting. complcte remissions can be induced. Inı J Cancer 1993:53:751-58.

89) Schöber C, Schulze M, Schlimok G, et al. Open label pilor study of monoclonal antibody 17-1 A in combination with 5-fluorouracil/levamisole or 5-FU/folinic acid in patients with advanced colorectal cancer [abstract]. Proc Am Soc Clin Oncol 1996:15:229.

90) Saleh MN, LoBuglio AF, Wheeler RH, et al. A phase Il trial of murine monoclonal antibody $17-1 \mathrm{~A}$ and interferong: clinical and immunological data. Cancer immunol Immunother 1990;32:185-90.

91) Wciner LM, Moldofsky P.J, Gatenby RA, et al. Antibody delivery and effector cell activation in a phase Il trial of recombinant g-interferon and the murine monoclonal anlibody CO17-1A in advanced coloreclal carcinoma. Cancer Res 1988;48:2568-73.

92) Weiner LM, Harvey E, Padavic-Shaller K, et al. Phase Il multicenter evaluation of prolonged murine monoclonal antibody 17-1 A therapy in pancreatic carcinoma. J Inmunother 1993;13:110-16.

93) Tempero MA, Sivinski C, Stcplewski Z, et al. Phase II trial of interieron gamma and monoclonal antibody $17-1 \mathrm{~A}$ in pancrealic cancer: biologic and clinical effects. J Clin Oncol 1990:8:2019-26.

94) Hempel D, Oru<io D, Müller P, et al. Transplantation of ex vivo tumor cell purged PBSCT in breast cancer paticnts treated with high dose chemotherapy followed by supplementary immunotherapy with MOAB 17-1 A phase I study [abstract). Proc Am Soc Clin Oncol 1998;17:145a.

95) Schmoll HJ, Quietzsch D, Börner P, et al. Safely of adjuvant mAb 17-I A in colorecial cancer (CRC) [Abstraci]. Eur J Cancer 1997;33 Suppl.8: S168. 